\title{
Phasic Changes in Bladder Compliance During Filling Cystometry of the Neurogenic Bladder
}

\author{
Soo-Yeon Kim, MD ${ }^{1,2,3}$, Sung Hwa Ko, MD ${ }^{1,2,3}$, Myung Jun Shin, MD ${ }^{1,2,4}$, Yeo Jin Park, MD ${ }^{1,2,4}$, \\ Ji Sang Park, $\mathrm{MD}^{1,2,3}$, Ko Eun Lee, $\mathrm{MD}^{2}$, Hyun-Yoon Ko, MD ${ }^{1,2,3}$
}

\begin{abstract}
${ }^{1}$ Division of Spinal Cord Medicine, ${ }^{2}$ Department of Rehabilitation Medicine, Pusan National University School of Medicine, Yangsan; ${ }^{3}$ Research Institute for Convergence of Biomedical Science and Technology, Pusan National University Yangsan Hospital, Yangsan; ${ }^{4}$ Medical Research Institute, Pusan National University, Busan, Korea
\end{abstract}

\begin{abstract}
Objective To investigate phasic changes during filling cystometry that most accurately represent detrusor properties, regardless of other factors affecting detrusor contractility.

Methods Seventy-eight patients (59 males, 19 females; mean age, 48.2 years) with spinal cord injuries were enrolled. Urodynamic studies were performed using a normal saline filling rate of $24 \mathrm{~mL} / \mathrm{min}$. We calculated bladder compliance values of the detrusor muscle in each of three filling phase intervals, which divided the filling cystometrogram into three phases referable to the cystometric capacity or maximum cystometric capacity. The three phases were sequentially delineated by reference to the pressure-volume curve reflecting bladder filling.

Results Bladder compliance during the first and second phases of filling cystometry was significantly correlated with overall bladder compliance in overactive detrusors. The highest coefficient of determination $\left(\mathrm{r}^{2}=0.329\right)$ was obtained during the first phase of the pressure-volume curve. Bladder compliance during all three phases was significantly correlated with overall bladder compliance of filling cystometry in underactive detrusors. However, the coefficient of determination was greatest $\left(\mathrm{r}^{2}=0.529\right)$ during the first phase of filling cystometry.

Conclusion Phasic bladder compliance during the early filling phase (first filling phase) was the most representative assessment of overall bladder compliance during filling cystometry. Careful determination of early phase filling is important when seeking to acquire reliable urodynamic data on neurogenic bladders.
\end{abstract}

Keywords Urodynamics, Compliance, Spinal cord injuries

Received December 2, 2013; Accepted January 29, 2014

Corresponding author: Hyun-Yoon Ko

Department of Rehabilitation Medicine, Pusan National University School of Medicine, 20 Geumo-ro, Yangsan 626-787, Korea

Tel: +82-55-360-4250, Fax: +82-55-360-4251, E-mail: drkohy@gmail.com

(a) This is an open-access article distributed under the terms of the Creative Commons Attribution Non-Commercial License (http://creativecommons. org/licenses/by-nc/3.0) which permits unrestricted noncommercial use, distribution, and reproduction in any medium, provided the original work is properly cited.

Copyright $\odot 2014$ by Korean Academy of Rehabilitation Medicine

\section{INTRODUCTION}

Diagnosis and effective management of neurogenic bladders require an evaluation of the function and condition of the lower urinary tract. Therefore, a urodynamic evaluation is necessary in addition to neurologic, physical, and radiographic assessments. A urodynamic evaluation includes filling cystometry, urethral pressure profiles, electromyography, and uroflowmetry. Filling 
cystometry records the pressure changes in bladder filling and measures reflex detrusor contraction of the neurogenic bladder and detrusor muscle tone $[1,2]$.

Bladder compliance is based on detrusor pressure at the start of bladder filling and corresponding bladder volume and detrusor pressure at cystometric capacity or immediately before the start of any detrusor contraction that causes significant leakage. This is defined as bladder volume changes in response to detrusor pressure changes during bladder filling, a measurement that reflects the histological changes and mechanical properties of the bladder [3,4].

As the bladder capacity reaches its limit, detrusor pressure increases gradually and a sense of voiding appears. However, in clinical practice, as the pressure-capacity curve during urodynamic evaluation often shows linear or irregular characteristics, bladder compliance is limited to reflect bladder status during the entire period of intravesical filling. These limitations of bladder compliance may be due to contracture of the detrusor muscles, loss of muscle fiber properties of the detrusor, or loss of mechanical viscoelasticity of the detrusors with post-void residuals. However, information on phasic changes during filling cystometry that influences bladder compliance is extremely limited. Thus, the present study measured bladder compliance during each filling phase (early, middle, and late) during a urodynamic evaluation, with the intention of determining the phase during filling cystometry that best reflects the condition of neurogenic bladders with both overactive and underactive or acon-

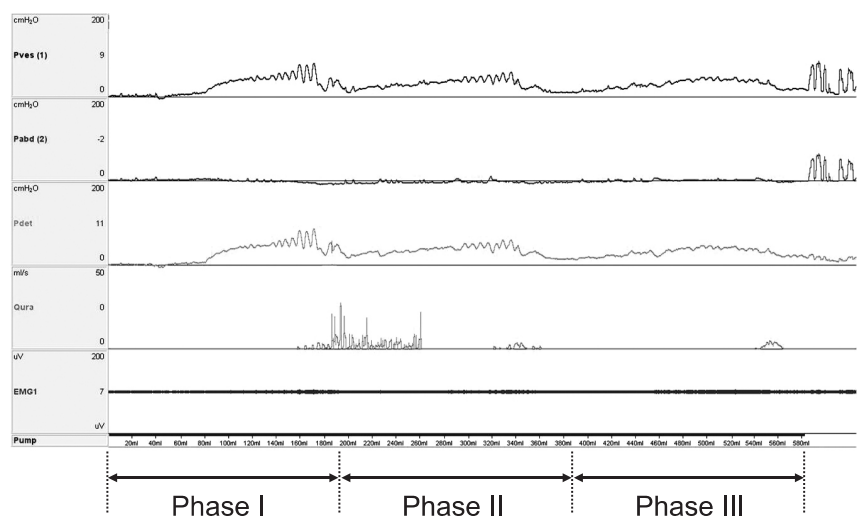

Fig. 1. Three phases of the filling cystometry. The bladder pressure-capacity curve was divided into three phases according to the points of filling, such as $1 / 3$ (phase I), $2 / 3$ (phase II), and 3/3 (phase III) of cystometric bladder capacity. tractile detrusors.

\section{MATERIALS AND METHODS}

The study cohort consisted of 78 patients (59 males, 19 females) with spinal cord injuries (mean period after injury, 43.4 months), divided into an overactive detrusor group (28 patients) and an underactive/acontractile detrusor group (50 patients).

Urodynamic evaluations were performed using Solar Gold (MMS, Enschede, The Netherlands). A saline solution was warmed to $32^{\circ} \mathrm{C}$ and infused into the bladder at a rate of $24 \mathrm{~mL} / \mathrm{min}$. Patients with an overactive detrusor were defined as those in which detrusor pressure increased involuntarily during the saline infusion, whereas patients with an underactive detrusor were defined as those with no increase in detrusor pressure or filling to $>600 \mathrm{~mL}$ without increased detrusor pressure [4].

To measure bladder compliance during each filling phase, the bladder pressure-capacity curve was divided into three filling cystometry phases according to the points of filling at $1 / 3$ (phase I), $2 / 3$ (phase II), and $3 / 3$ (phase III) of cystometric bladder capacity (Fig. 1). The characteristics of each filling cystometry phase were examined by measuring bladder compliance of entire filling cystometry relative to the change in detrusor pressure during each phase.

Statistical analyses were performed using SPSS ver. 10.0 (SPSS Inc., Chicago, IL, USA). Pearson correlation test was used to compare differences between bladder compliance during each filling phase and bladder compliance during the entire filling cystometry test. Statistical significance was set at $\mathrm{p}<0.05$.

Table 1. Demographic and clinical characteristics of the subjects

\begin{tabular}{lcc}
\hline \multicolumn{1}{c}{ Characteristic } & $\begin{array}{c}\text { Overactive } \\
\text { detrusor } \\
(\mathbf{n = 2 8})\end{array}$ & $\begin{array}{c}\text { Underactive } \\
\text { detrusor } \\
(\mathbf{n = 5 0 )}\end{array}$ \\
\hline Sex (male:female) & $21: 7$ & $38: 12$ \\
Age (yr) & $42.9 \pm 15.6$ & $51.4 \pm 16.1$ \\
Time after injury (mo) & $52.0 \pm 42.0$ & $39.3 \pm 63.1$ \\
Level of injury & & \\
Cervical & 19 & 19 \\
Thoracic & 8 & 22 \\
Lumbar & 1 & 9 \\
\hline
\end{tabular}




\section{RESULTS}

Twenty-eight patients ( 21 males, 7 females) were included in the overactive detrusor group. The mean age of these patients was 42.9 years and the mean time interval after spinal cord injury was 52.0 months. Their neurological levels of injury were the cervical cord in 19, the thoracic cord in eight, and the lumbar cord in one. Fifty patients (38 males, 12 females) were included in the underactive detrusor group. Their mean age was 51.4 years and the mean period after injury was 39.3 months. Their neurological levels of injury were the cervical cord in 19, the thoracic cord in 22, and the lumbar cord in nine (Table 1).

Bladder compliance during the entire filling cystometry test was significantly lower in the overactive than that in the underactive detrusor group ( $37.5 \pm 24.9$ vs. $56.8 \pm 45.2$ $\left.\mathrm{mL} / \mathrm{cm} \mathrm{H}_{2} \mathrm{O} ; \mathrm{p}<0.05\right)$. Bladder compliance values during phases I, II, and III in the overactive detrusor group were $31.6 \pm 20.5,44.3 \pm 39.3$, and $36.2 \pm 72.4 \mathrm{~mL} / \mathrm{cm} \mathrm{H}_{2} \mathrm{O}$, respectively. Bladder compliance values during phases I and II were each significantly correlated with bladder compliance during the entire filling cystometry test with the highest coefficient of determination during phase I $\left(r^{2}=0.329\right)$ (Table 2). In the underactive detrusor group, bladder compliance values during phases I, II, and III were $69.6 \pm 62.3,109.4 \pm 73.4$, and $56.2 \pm 59.3 \mathrm{~mL} / \mathrm{cm} \mathrm{H}_{2} \mathrm{O}$, respectively, and all showed significant correlations with compliance during the entire filling phase. The coefficient of determination was highest during phase I $\left(r^{2}=0.529\right)$ (Table 3).

Table 2. Bladder compliance during different phases of filling cystometry in 28 patients with an overactive detrusor

\begin{tabular}{|c|c|c|c|}
\hline & $\begin{array}{l}\text { Compliance } \\
\left(\mathrm{mL} / \mathrm{cm} \mathrm{H}_{2} \mathrm{O}\right)\end{array}$ & p-value & $\mathbf{r}^{2}$ \\
\hline Total filling phase & $37.5 \pm 24.9$ & - & - \\
\hline \multicolumn{4}{|l|}{ Phase $^{\text {a) }}$} \\
\hline I & $31.6 \pm 20.5$ & $0.002^{*}$ & 0.329 \\
\hline II & $44.3 \pm 39.3$ & $0.030^{*}$ & 0.168 \\
\hline III & $36.2 \pm 72.4$ & 0.830 & 0.001 \\
\hline
\end{tabular}

Comparison of compliances between each phase and total filling phase.

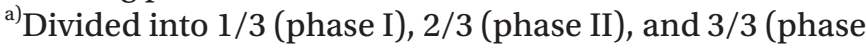
III) of cystometric bladder capacity. ${ }^{*} \mathrm{p}<0.05$.

\section{DISCUSSION}

We measured bladder compliance during each phase (early, middle, and late) of a filling cystometry during a urodynamic evaluation. We determined the phase during filling cystometry that best reflected the condition of neurogenic bladders with both overactive and underactive detrusors. Bladder compliance is limited in reflecting bladder status during the entire period of intravesical filling due to mechanical changes in the detrusor muscles, including contracture of the detrusor muscles, loss of muscle fiber properties of the detrusor, and loss of viscoelasticity of the detrusors with post-void residuals in a chronic neurogenic bladder [1]. We have proposed an alternative measurement of bladder compliance, which considers multiple phases of the entire filling cystometry.

Bladder function has been evaluated by measuring the point of bladder filling sense, bladder volume, detrusor activities, and detrusor compliance. The sympathetic nerve in the bladder is excited during the urine storage phase. As bladder capacity increases, receptors in the bladder mucosa are stimulated through excitation of the pelvic nerve, and spinal reflexes are activated $[1,5]$. As infusion of saline begins during a urodynamic evaluation, the initial reactions of the myogenic and viscoelastic elements of the bladder occur, following a constant pressure for inflation of bladder capacity. Sudden pressure increases or involuntary detrusor contractions occur at the limit of bladder viscoelasticity. At the onset of filling, the pressure-volume curve is determined primarily by the properties of the muscular wall [6], whereas later filling is

Table 3. Bladder compliance during different phases of filling cystometry in 50 patients with an underactive detrusor

\begin{tabular}{|c|c|c|c|}
\hline & $\begin{array}{c}\text { Compliance } \\
\left(\mathrm{mL} / \mathrm{cm} \mathrm{H}_{2} \mathrm{O}\right)\end{array}$ & p-value & $\mathbf{r}^{2}$ \\
\hline Total filling phase & $56.8 \pm 45.2$ & - & - \\
\hline \multicolumn{4}{|l|}{ Phase $^{\text {a) }}$} \\
\hline I & $69.6 \pm 62.3$ & $<0.001^{*}$ & 0.529 \\
\hline II & $109.4 \pm 73.4$ & $<0.001^{*}$ & 0.421 \\
\hline III & $56.2 \pm 59.3$ & $<0.001^{*}$ & 0.345 \\
\hline
\end{tabular}

Comparison of compliances between each phase and total filling phase.

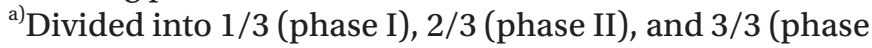
III) of cystometric bladder capacity. ${ }^{*} \mathrm{p}<0.05$. 
registered neurologically and regulated by spinal reflexes $[1,7]$.

From a urodynamic standpoint, we found that the early filling phase provided more information about bladder response during filling than any other filling phase in both groups of overactive and underactive or acontractile detrusors.

In overactive detrusor, initial reactions of the myogenic and viscoelastic elements of the bladder as alterations in the connective tissue of the bladder wall [2] may be reflected by a correlation between the early phase of bladder compliance and bladder compliance of the entire filling cystometry test in this study.

The theory of detrusor underactivity postulates that the decrease in detrusor contraction is caused by an increase of collagen in the detrusor and a decrease in receptors or the ion channels [8]. This is followed by a decrease in the stimulus that causes micturition reflex activation due to sensory dysfunction and a decrease in efferent stimulation of the detrusor. The correlation between the early phase of bladder compliance and bladder compliance of the entire filling cystometry in individuals with an underactive detrusor may be due to the definition of the overactive detrusor itself, which is based on any overactivity of the detrusor during the filling phase. Thus, changes in bladder elasticity rather than changes in the contractile function of the detrusor will affect changes in compliance during the early filling phase.

Bladder compliance is determined by various elements, including those related to technique, filling rate, filling medium properties, and pressure and capacity changes [1]. The 2002 International Continence Society (ICS) definition [4] states that bladder compliance can be calculated by dividing the change in volume by the change in detrusor pressure. Two standard points should be used: the start of filling and cystometric capacity, or immediately before any detrusor contractions that will end the test, for example, due to significant leakage $[1,4]$. Theoretically, the best filling rate would be achieved by physiological natural filling. The ICS 2002 standardized filling rates define slow filling as $<10 \mathrm{~mL} / \mathrm{min}$, medium filling as $10-100$ $\mathrm{mL} / \mathrm{min}$, and rapid filling as $>100 \mathrm{~mL} / \mathrm{min}$. In clinical practice, filling rates in adults, range from 10 to $100 \mathrm{ml} /$ min, with most being $30-50 \mathrm{~mL} / \mathrm{min}[1,6]$. Thus, the current filling rate of $24 \mathrm{~mL} / \mathrm{min}$ likely underestimated true compliance.
Detrusor changes depend on the neurological level of the spinal cord injury [9]. For example, detrusor hyperreflexia and detrusor sphincter dyssynergia have often been observed in patients with cervical cord injury, detrusor hyperreflexia predominates in patients with thoracic cord injury, and detrusor areflexia is often observed in patients with lumbosacral cord injury. However, we found that detrusor activities were not determined by the neurological level of the spinal cord injury. In fact, many patients in the underactive detrusor group had cervical and thoracic spinal cord injuries. However, the percentage of patients with thoracic and lumbar cord injuries was higher in the underactive than those in the overactive detrusor group. Because the mechanisms underlying spinal cord injuries vary, urodynamics may not correlate with the level of spinal cord injury. In several patients with cervical and thoracic spinal cord injuries, the detrusor reflex conditions changed due to placement of the urethral catheter, histological changes in the bladder due to frequent infections and inflammation, or long-term use of bladder medication.

The limitations of this study are as follows: 1) patients with a spinal cord injury and who were taking medication (anticholinergics or cholinergics) were included. The drug effect could have influenced the parameters of the urodynamic study. And 2) constant bladder filling rates of $24 \mathrm{~mL} / \mathrm{min}$ in the urodynamic study likely underestimated true compliance in overactive detrusors and overestimated true compliance in underactive or acontractile detrusors. However, a constant bladder filling rate has the benefit to obtain regulated data from the same parameters.

In conclusion, we compared bladder compliance during each phase of filling cystometry with detrusor compliance during the entire filling cystometry using urodynamic assessments of neurogenic bladders. Phasic compliance during the early filling phase was the most representative means of assessing bladder compliance measured during entire filling cystometry, regardless of detrusor activity. Thus, from a urodynamic standpoint, the early filling phase provides more information about bladder response than any other filling phase during filling cystometry. Careful assessment of early phase filling is important when determining reliable urodynamic data on neurogenic bladders. 


\section{CONFLICT OF INTEREST}

No potential conflict of interest relevant to this article was reported.

\section{ACKNOWLEGMENTS}

This work was partly supported by a 2-year research grant of Pusan National University.

\section{REFERENCES}

1. Wyndaele JJ, Gammie A, Bruschini H, De Wachter S, Fry $\mathrm{CH}$, Jabr RI, et al. Bladder compliance what does it represent: can we measure it, and is it clinically relevant? Neurourol Urodyn 2011;30:714-22.

2. Landau EH, Jayanthi VR, Churchill BM, Shapiro E, Gilmour RF, Khoury AE, et al. Loss of elasticity in dysfunctional bladders: urodynamic and histochemical correlation. J Urol 1994;152(2 Pt 2):702-5.

3. Abrams P. Urodynamics. 3rd ed. London: Springer;
2006.

4. Abrams P, Cardozo L, Fall M, Griffiths D, Rosier P, Ulmsten $\mathrm{U}$, et al. The standardisation of terminology of lower urinary tract function: report from the Standardisation Sub-committee of the International Continence Society. Neurourol Urodyn 2002;21:167-78.

5. CSAPO AI. Smooth muscle as a contractile unit. Physiol Rev Suppl 1962;5:7-33.

6. Edvardsen P. Nervous control of urinary bladder in cats. I. The collecting phase. Acta Physiol Scand 1968;72:157-71.

7. Marsh DJ, Suzuki G, Meyers FH. Role of afferent activity from the bladder in regulating its activity. Am J Physiol 1959;196:351-3.

8. van Koeveringe GA, Vahabi B, Andersson KE, Kirschner-Herrmans R, Oelke M. Detrusor underactivity: a plea for new approaches to a common bladder dysfunction. Neurourol Urodyn 2011;30:723-8.

9. Kaplan SA, Chancellor MB, Blaivas JG. Bladder and sphincter behavior in patients with spinal cord lesions. J Urol 1991;146:113-7. 